Fanum

Sociológico
Forum Sociológico

Série II

$22 \mid 2012$

Saúde e multiculturalidade

\title{
Encontro com a diferença: a perspetiva dos profissionais de saúde no contexto da prestação de cuidados aos imigrantes
}

Rita Rodrigues e Sónia Dias

\section{(2) OpenEdition \\ Journals}

Edição electrónica

URL: https://journals.openedition.org/sociologico/583

DOI: $10.4000 /$ sociologico.583

ISSN: 2182-7427

Editora

CICS.NOVA - Centro Interdisciplinar de Ciências Sociais da Universidade Nova de Lisboa

Edição impressa

Paginação: 63-72

ISSN: 0872-8380

Refêrencia eletrónica

Rita Rodrigues e Sónia Dias, «Encontro com a diferença: a perspetiva dos profissionais de saúde no contexto da prestação de cuidados aos imigrantes», Forum Sociológico [Online], 22 | 2012, posto online no dia 26 fevereiro 2013, consultado o 30 março 2022. URL: http://journals.openedition.org/ sociologico/583 ; DOI: https://doi.org/10.4000/sociologico.583

Este documento foi criado de forma automática no dia 30 março 2022.

(c) CICS.NOVA 


\title{
Encontro com a diferença: a perspetiva dos profissionais de saúde no contexto da prestação de cuidados aos imigrantes ${ }^{1}$
}

\author{
Rita Rodrigues e Sónia Dias
}

\section{Introdução}

1 O aumento da intensidade dos movimentos migratórios tem conduzido a um processo contínuo de transformação social, económica e demográfica nas sociedades atuais (Davies et al., 2009).

2 A saúde apresenta-se como um eixo fundamental no quadro dos processos de integração, sendo considerada como condição essencial para o desenvolvimento das sociedades (Machado, 2007). A migração, enquanto fenómeno social complexo, constitui um importante desafio nas dinâmicas dos serviços de saúde e na prestação de cuidados de saúde nos países de acolhimento (Peiro e Benedict, 2009). Neste contexto, emerge a necessidade de diferenciação das práticas no domínio da prestação de cuidados e das condições de acesso aos serviços de saúde face a uma população cujas características se têm vindo a modificar dada a profusão de utentes de diversas origens.

3 As perceções e atitudes dos profissionais de saúde no contexto da prestação de cuidados podem ter implicações na sua interação, facto que se pode refletir no atendimento e na prática clínica, em particular, ao nível do diagnóstico e da prescrição de tratamentos, assim como na própria procura dos serviços de saúde por parte dos imigrantes (Betancourt et al., 2003; Campayo et al., 2006; Eshiett e Parry, 2003; Scheppers et al., 2006). A postura que os profissionais de saúde assumem no âmbito do atendimento e da prestação de cuidados é indissociável do quadro sociocultural no qual estão inseridos (Van Ryn e Burke, 2000), o que significa que a experiência e visão do mundo se refletem nas representações, atitudes e práticas quotidianas. O contexto relacional é não só 
estruturado a partir das atitudes dos profissionais de saúde, como também mediante as próprias características do sistema de saúde e as especificidades dos utentes.

Adicionalmente, tem vindo a ser reconhecido que os profissionais de saúde enfrentam diversas dificuldades no atendimento e na prestação de cuidados de saúde a imigrantes (Priebe et al., 2011), situação que vem colocar novos desafios à estrutura e organização dos serviços de saúde, assim como à prática clínica (Dias, Rodrigues et al., 2010).

O estudo das atitudes dos profissionais de saúde perante a diversidade cultural permite compreender como estes assimilam a experiência do contacto com a diferença e as repercussões na relação entre profissional e utente. A escassez de informação sobre esta temática ao nível internacional e nacional (Fonseca et al., 2009; Ingleby et al., 2005), e o crescente enfoque na necessidade de adaptar os serviços de saúde suscitou o interesse em explorar perceções e atitudes, bem como em compreender melhor os desafios que os profissionais enfrentam no atendimento e na prestação de cuidados de saúde a utentes imigrantes.

6 Pretende-se com este trabalho descrever e compreender as perceções e atitudes dos profissionais de saúde no contexto da prestação de cuidados de saúde a imigrantes africanos, brasileiros e dos Países da Europa de Leste.

\section{Metodologia}

7 Para a concretização dos objetivos propostos foram desenvolvidos dois estudos, um estudo assente numa abordagem quantitativa e um numa abordagem qualitativa, as quais "[...] representam um continuum epistemológico e não uma dicotomia" (LessardHérbert et al., 1994: 35).

Os participantes dos estudos consistiram em médicos, enfermeiros e administrativos que exercem funções em Centros de Saúde, uma vez que estes representam a estrutura de primeira linha de cuidados de saúde ao indivíduo. A seleção dos participantes foi realizada com base no seguinte critério de inclusão: profissionais que exercem funções nos serviços de cuidados de saúde primários da região de Lisboa e Vale do Tejo.

Os estudos foram aprovados pelo Conselho de Ética do Instituto de Higiene e Medicina Tropical da Universidade Nova de Lisboa.

\section{Estudo quantitativo}

Realizou-se um estudo descritivo e transversal com uma amostra representativa de 400 profissionais de saúde. Do total da amostra, 320 profissionais aceitaram participar no estudo, dos quais 115 são administrativos, 114 enfermeiros e 91 médicos. A maioria dos participantes é do sexo feminino $(84,1 \%)$ e $15,9 \%$ do sexo masculino. Relativamente à faixa etária, cerca de $58 \%$ dos profissionais tem 45 anos ou mais.

11 A amostra foi selecionada através da realização de um processo de amostragem aleatório simples de 40 Centros de Saúde e um processo de amostragem aleatório simples de 10 profissionais de saúde de cada Centro de Saúde. Os dados foram recolhidos através de um inquérito por questionário de autopreenchimento que incluiu questões relativas à caracterização sociodemográfica dos participantes e à perceção sobre imigração, saúde e doença. O segundo conjunto de questões foi composto por 
perguntas de resposta fechada com uma escala de concordância tipo Likert (e. g., discordo, não concordo nem discordo e concordo).

O questionário foi submetido a um pré-teste através da realização de grupos focais de modo a garantir a sua adequação quanto ao conteúdo, formulação, ordenação das perguntas e duração da aplicação.

13 A recolha de dados decorreu entre os meses de Outubro de 2008 e Fevereiro de 2009. Os centros de saúde foram contactados pela equipa de investigação com o objetivo de apresentar o estudo e solicitar autorização para a sua realização. Em cada centro de saúde, os questionários foram distribuídos aos profissionais que os devolviam em envelopes fechados para que, posteriormente, fossem reenviados à equipa de investigação.

Os questionários foram inseridos numa base de dados através de um programa de leitura ótica. Posteriormente, os dados foram transferidos para uma base de dados de SPSS 17.0 (Statistical Package for Social Sciences) para se proceder ao tratamento estatístico e à análise descritiva das variáveis em estudo.

\section{Estudo qualitativo}

15 No âmbito do estudo qualitativo procedeu-se à recolha de dados através da técnica de grupos focais ou grupos de discussão centrados num tema (Morgan, 2001). 0 grupo focal é um instrumento de natureza qualitativa que nos permite captar os significados e as posições individuais de modo a compreender como estas se relacionam com as perspetivas do grupo, permitindo a obtenção de dados mais aprofundados e detalhados acerca do objeto de estudo (Krueger e Casey, 2000).

16 Neste estudo foi utilizada uma amostra intencional de 32 profissionais de saúde dos serviços de cuidados de saúde primários que foram contactados pela equipa de investigação em colaboração com a Administração Regional de Saúde. Realizaram-se três grupos focais, um com cada grupo de profissionais, nomeadamente médicos, enfermeiros e administrativos.

17 Os grupos focais decorreram entre Março e Abril de 2010. Cada grupo focal teve uma duração média de uma hora e trinta minutos, sendo que no início de cada sessão foi apresentada informação sobre os objetivos do estudo e o modo de condução das sessões. Cada sessão foi registada em áudio, com o consentimento prévio dos participantes, no sentido de otimizar a recolha de dados e facilitar a transcrição das discussões realizadas para análise posterior. 0 consentimento escrito voluntário dos participantes foi obtido com garantia do seu anonimato e da confidencialidade dos dados.

18 Para a condução dos grupos focais foi elaborado um guião semiestruturado, previamente organizado, com os seguintes tópicos a explorar: conhecimentos e competências na área da saúde dos imigrantes, perceção sobre a saúde e doença nos imigrantes, e o atendimento e a prestação de cuidados de saúde à população imigrante.

Os dados foram analisados de acordo com o procedimento da análise de conteúdo (Bardin, 2007), identificando-se os temas emergentes. 0 discurso dos participantes foi estruturado em dimensões, subdivididas em categorias de análise que passaram por um processo de (re)ajustamento à medida que decorria a análise dos dados. Analisaram-se as opiniões sobre os vários temas, considerando os consensos gerados nos grupos e as contribuições individuais. 
20 De modo a manter a confidencialidade dos dados, todos os participantes são identificados neste trabalho como Médicos, Enfermeiros e Administrativos.

\section{Perceção sobre o utente imigrante (Quadro 1)}

21 Quando questionados sobre a perceção relativamente ao imigrante enquanto utente, $40,2 \%$ dos participantes discordou de que os imigrantes não respeitam as normas de funcionamento dos serviços de saúde. Embora 47,7\% dos médicos e 46,7\% dos enfermeiros tenham discordado, $48,1 \%$ de administrativos concordaram com esta afirmação $(p=0,004)$.

Quadro 1 臨 Perceção sobre o utente imigrante

\begin{tabular}{|c|c|c|c|c|c|}
\hline \multirow{2}{*}{ Os imigrantes: } & Total & Administrativos & Enfermeiros & Médicos & $p$ \\
\hline & $\%$ & $\%$ & $\%$ & $\%$ & \\
\hline $\begin{array}{c}\text { Não respeitam as normas de } \\
\text { funcionamento dos serviços de saúde }(n=301)\end{array}$ & & & & & \\
\hline Discordo & 40,2 & 27,8 & 46,7 & 47,7 & \multirow{3}{*}{$\begin{array}{l}0,00 \\
4\end{array}$} \\
\hline Não Concordo nem Discordo & 24,6 & 24,1 & 27,1 & 22,1 & \\
\hline Concordo & 35,2 & 48,1 & 26,2 & 30,2 & \\
\hline \multicolumn{6}{|l|}{ Comportam-se como vítimas ( $n=296$ ) } \\
\hline Discordo & 61,8 & 47,3 & 63,9 & 77,5 & \multirow{3}{*}{$\begin{array}{l}<0,0 \\
01\end{array}$} \\
\hline Não Concordo nem Discordo & 19,3 & 22,7 & 20,6 & 13,5 & \\
\hline Concordo & 18,9 & 30,0 & 15,5 & 9,0 & \\
\hline \multicolumn{6}{|l|}{$\begin{array}{l}\text { São mais reivindicativos do que a } \\
\text { população } \\
\text { em geral }(n=304)\end{array}$} \\
\hline Discordo & 36,8 & 30,4 & 43,4 & 37,2 & \multirow{3}{*}{$\begin{array}{l}0,00 \\
2\end{array}$} \\
\hline Não Concordo nem Discordo & 28,0 & 20,5 & 33,0 & 31,4 & \\
\hline Concordo & 35,2 & 49,1 & 23,6 & 31,4 & \\
\hline \multicolumn{6}{|l|}{ São dificeis, agressivos e perigosos $(n=308)$} \\
\hline Discordo & 19,5 & 13,0 & 27,3 & 17,8 & \multirow{3}{*}{0,007} \\
\hline Não Concordo nem Discordo & 30,8 & 26,8 & 36,4 & 28,9 & \\
\hline Concordo & 49,7 & 60,2 & 36,4 & 53,3 & \\
\hline
\end{tabular}

22 A maioria dos profissionais discordou de que os imigrantes comportam-se como vítimas (61,8\%). No entanto, os administrativos apresentaram maior grau de concordância com esta afirmação (30\%) do que se verifica no grupo dos médicos e enfermeiros $(9,0 \%$ e $15,5 \%$, respectivamente) $(p=<0,001)$. Do total dos participantes, $36,8 \%$ discordaram de que os imigrantes são mais reivindicativos do que a população em geral, enquanto $35,2 \%$ concordaram com esta afirmação. Verificam-se diferenças significativas entre os grupos profissionais, sendo que $49,1 \%$ dos administrativos concordaram, enquanto $37,2 \%$ dos médicos e $43,4 \%$ dos enfermeiros discordaram $(p=0,002)$.

Cerca de $50 \%$ dos profissionais consideraram que os imigrantes são difíceis, agressivos e perigosos, verificando-se que 60,2\% dos administrativos e 53,3\% dos médicos concordaram. No entanto, as respostas dos enfermeiros dividem-se em ambas as categorias "não concordo nem discordo" e "concordo" de igual forma $(36,4 \%)(p=0,007)$. 


\section{Atitudes perante a imigração, saúde e doença (Quadro 2)}

No que se refere às atitudes perante a imigração, $64,3 \%$ consideraram que as sociedades que acolhem os imigrantes tornam-se mais solidárias e culturalmente ricas e 61,6\% referiram que os imigrantes são importantes porque contribuem para a economia e desenvolvimento do país. Não se verificam diferenças significativas entre os grupos profissionais.

Por outro lado, uma grande proporção de profissionais tende a concordar que o aumento do número de imigrantes em Portugal pode ser uma ameaça para o país (39,5\%). Verificam-se diferenças entre os grupos, pois $49,1 \%$ dos administrativos e $39,6 \%$ dos enfermeiros concordaram com a afirmação, enquanto $37,8 \%$ dos médicos discordaram $(p=0,020)$.

Quadro 2 踇 Atitudes perante a imigração, saúde e doença

\begin{tabular}{|c|c|c|c|c|c|}
\hline & Total & Administrativos & Enfermeiros & Médicos & $p$ \\
\hline & $\%$ & $\%$ & $\%$ & $\%$ & \\
\hline \multicolumn{6}{|c|}{$\begin{array}{l}\text { As sociedades que acolhem imigrantes tornam-se } \\
\text { mais solidárias e mais ricas culturalmente }(n=305)\end{array}$} \\
\hline Discordo & 7,2 & 10,3 & 5,5 & 5,7 & \multirow{3}{*}{0,498} \\
\hline Não Concordo nem Discordo & 28,5 & 30,8 & 28,4 & 25,8 & \\
\hline Concordo & 64,3 & 58,9 & 66,1 & 68,5 & \\
\hline \multicolumn{6}{|c|}{$\begin{array}{l}\text { Os imigrantes são importantes porque contribuem } \\
\text { para a economia e o desenvolvimento do pais ( } n=305 \text { ) }\end{array}$} \\
\hline Discordo & 4,6 & 3,7 & 5,6 & 4,4 & \multirow{3}{*}{0,261} \\
\hline Não Concordo nem Discordo & 33,8 & 34,6 & 39,8 & 25,6 & \\
\hline Concordo & 61,6 & 61,7 & 54,6 & 70,0 & \\
\hline \multicolumn{6}{|c|}{$\begin{array}{l}\text { O aumento do número de imigrantes em Portugal pode } \\
\text { ser uma ameaça para o pais }(n=309)\end{array}$} \\
\hline Discordo & 27,8 & 19,4 & 27,9 & 37,8 & \multirow{3}{*}{0,020} \\
\hline Não Concordo nem Discordo & 32,7 & 31,5 & 32,4 & 34,4 & \\
\hline Concordo & 39,5 & 49,1 & 39,6 & 27,8 & \\
\hline \multicolumn{6}{|c|}{$\begin{array}{l}\text { O estado de saúde dos imigrantes depende da sua } \\
\text { integração no país de acolhimento }(n=302)\end{array}$} \\
\hline Discordo & 12,6 & 20,8 & 13,1 & 2,3 & \multirow{3}{*}{0,001} \\
\hline Não Concordo nem Discordo & 18,9 & 20,8 & 19,6 & 15,7 & \\
\hline Concordo & 68,5 & 58,4 & 67,3 & 82,0 & \\
\hline \multicolumn{6}{|c|}{$\begin{array}{l}\text { A imigração acarreta dificuldades que potenciam o } \\
\text { aparecimento de doenças }(n=297)\end{array}$} \\
\hline Discordo & 13,8 & 9,7 & 13,8 & 16,2 & \multirow{3}{*}{0,143} \\
\hline Não Concordo nem Discordo & 27,3 & 35,9 & 27,3 & 21,9 & \\
\hline Concordo & 58,9 & 54,4 & 58,9 & 61,9 & \\
\hline \multicolumn{6}{|c|}{$\begin{array}{l}\text { As doenças nos imigrantes são um perigo para a saúde } \\
\text { pública }(n=300)\end{array}$} \\
\hline Discordo & 45,7 & 42,5 & 42,9 & 52,8 & \multirow{3}{*}{0,622} \\
\hline Não Concordo nem Discordo & 38,7 & 40,5 & 41,0 & 33,7 & \\
\hline Concordo & 15,6 & 17,0 & 16,1 & 13,5 & \\
\hline
\end{tabular}

No que se refere à perceção sobre imigração e saúde, $68,5 \%$ dos profissionais concordaram que o estado de saúde dos imigrantes depende da sua integração no país de acolhimento. No entanto, uma maior proporção de administrativos $(20,8 \%)$ discordou, comparativamente com $13,1 \%$ de enfermeiros e $2,3 \%$ de médicos $(p=0,001)$. As respostas distribuem-se de forma semelhante nos três grupos profissionais.

Relativamente à perceção sobre imigração e doença, 58,9\% referiram que a imigração acarreta dificuldades que potenciam o aparecimento de doenças. Do conjunto de profissionais de saúde, 45,7\% discordaram que as doenças nos imigrantes são um perigo para a saúde pública, no entanto cerca de $16 \%$ concordaram com esta afirmação. Não se verificam diferenças significativas entre os grupos profissionais. 


\section{Grupos focais}

\section{Perceção sobre o utente imigrante}

$28 \mathrm{Na}$ opinião de alguns participantes, os imigrantes não podem ser considerados como um grupo socialmente homogéneo, verificando-se diferenças entre indivíduos oriundos de diversas zonas geográficas, e ainda entre indivíduos com a mesma nacionalidade e/ ou pertença étnica: “(...) são muito diferentes. É impossível caracterizar um imigrante" (Médico). No entanto, alguns enfermeiros consideram que, no geral, os imigrantes são pouco reivindicativos, embora esta opinião não se tenha revelado unânime: "Eu diria que pouco reivindicativos, acabam por aceitar..."; "Depende de onde vêm" (Enfermeiros).

\section{Perceção sobre a saúde e doença nos imigrantes}

A maioria dos enfermeiros considerou que, muitas vezes, a perceção dos profissionais sobre os problemas de saúde dos imigrantes está associada a estereótipos e preconceitos que possuem em relação a estas populações: “(...) a diferença está no nosso olhar. Eu tenho portugueses que têm características idênticas às dos imigrantes (...) e imigrantes que se calhar têm características iguais aos outros... cada caso é um caso" (Enfermeiro). Neste sentido, vários enfermeiros reforçaram que, no geral, os problemas de saúde nos imigrantes não diferem da população autóctone: “(...) o binómio saúde/doença para o imigrante é igual para o português" (Enfermeiro).

Quando questionados sobre a saúde e doença na população imigrante, os enfermeiros e administrativos salientaram que a vulnerabilidade em saúde dos imigrantes se encontra associada às condições socioeconómicas precárias e a situações de exclusão social: “... nós sabemos que a pobreza efetivamente está ligada à saúde. Sabemos que os imigrantes quando vêm, habitualmente vivem em condições precárias" (Enfermeiro). No entanto, alguns médicos consideram que, de modo geral, a população imigrante é saudável.

Alguns médicos e enfermeiros consideram que a doença nos imigrantes pode representar um risco em termos de saúde pública: “Os imigrantes recém-chegados (...) são doenças a entrar no país, são pessoas doentes a entrar. Muitas delas vêm até só por motivos de doença para tratamento...” (Médico); “... aqueles que não nos procuram serão se calhar o maior risco em termos de saúde pública. Para eles e para nós enquanto cidadãos portugueses" (Enfermeiro).

\section{Perceção sobre as atitudes dos profissionais de saúde perante o utente imigrante}

Vários participantes consideram que as atitudes dos profissionais podem criar dificuldades no atendimento e na prestação de cuidados de saúde: "Acho que os médicos, mais do que os enfermeiros, podem eventualmente criar mais problemas. Até porque os enfermeiros têm um contacto mais próximo" (Médicos); "[os médicos] no fundo não se querem envolver" (Enfermeiro).

Os participantes também descreveram a existência de situações de discriminação e de estigmatização por parte dos profissionais de saúde: "Não sei se os imigrantes não serão tratados um bocadinho como de segunda... Estão mais vulneráveis" (Médico); Assisto ao preconceito por parte dos enfermeiros no atendimento a estas pessoas" (Enfermeiro); "Há 
pessoas que estão renitentes aos migrantes (...) racismo a todos os níveis dos profissionais, colegas nossos" (Administrativo).

\section{Desafios no contexto da prestação de cuidados de saúde a imigrantes}

Os profissionais descreveram um conjunto de dificuldades que estes enfrentam no atendimento de utentes imigrantes tendo implicações na prestação de cuidados de saúde. Os participantes salientaram dificuldades de comunicação devido a diferenças linguísticas: "Ao nível de consultas o que acontece frequentemente é a barreira da linguagem" (Médico); "A linguagem é uma barreira imensa" (Enfermeiro); "Não nos percebem e nós não os percebemos a eles. Temos vários problemas por causa disso" (Administrativo).

A falta de prática no diagnóstico e tratamento de doenças tropicais ou invulgares foi descrita pelos médicos como uma dificuldade à prática clínica, salientando a necessidade de encaminhamento para serviços especializados, nomeadamente para o Instituto de Higiene e Medicina Tropical: "Em termos de patologias, por exemplo. Já houve sinalização de casos de lepra em brasileiros de que eu não tinha ideia" (Médico).

Os profissionais descreveram como as diferenças culturais interferem no processo de diagnóstico e na prescrição de tratamentos e como o desconhecimento das práticas culturais e religiosas constitui uma dificuldade no âmbito da prestação de cuidados: "Nós é que ficamos à nora porque não sabemos nada das crenças, nem da cultura que eles têm" (Médico); "O nosso desconhecimento da base cultural vai no fundo estar sempre muito presente na avaliação que fazemos e não sei até que ponto é que nós próprios não prejudicaremos, por vezes, os utentes" (Enfermeiro); "Temos aquelas situações em que elas andam de burka, que para as auscultar não querem sequer às vezes destapar-se." (Administrativo).

Neste contexto, alguns participantes referem que os profissionais possuem reduzidas capacidades para lidar com a diversidade cultural, o que pode interferir na relação entre profissional e utente e, em simultâneo, impor-se como um obstáculo à continuidade na procura de cuidados de saúde pelos imigrantes: "Temos a situação daqueles que prestam os cuidados mas que por falta de conhecimento impõem aquilo que são as nossas regras, os nossos costumes, isso não é por si só a barreira, mas é uma dificuldade que se cria à continuidade do acesso daqueles imigrantes aos serviços de saúde" (Enfermeiro).

De acordo com os participantes, a falta de sensibilidade perante as diferenças culturais por parte dos profissionais pode contribuir para a existência de dificuldades no relacionamento entre profissional e utente: "Do ponto de vista cultural há algumas dificuldades de entender, de querer entender, de arranjar subterfúgios para superar dificuldades" (Médico); "Preocupa-me a falta de sensibilidade dos profissionais de saúde para as questões das diferenças culturais (...)” (Enfermeiro); "Nem estão [profissionais de saúde] sensibilizados para tal. Nem estamos para os nossos, quanto mais para os outros [imigrantes]!" (Administrativo).

Neste contexto, foi ainda referido como as questões de género podem dificultar a prestação de cuidados de saúde: "Dizer a um muçulmano para se despir para mostrar o problemazinho que tem no pénis, a ver se ele se despe ao pé de ti. Nem pensar!" (Médico). 


\section{Discussão}

40 A perceção dos profissionais de saúde face aos imigrantes evidencia uma visão geral da imigração enquanto fenómeno que contribui de modo positivo para a sociedade. No entanto, aferimos que a maior parte dos participantes considera que o aumento do número de imigrantes pode ser uma ameaça para o país, reiterando os resultados do estudo realizado por Lages e colaboradores (2006) sobre as atitudes e os valores da população portuguesa perante a imigração e os imigrantes. De acordo com Lages et al. (2006), apesar das perceções positivas sobre a imigração e os imigrantes, a maioria dos portugueses considera que o fluxo imigratório em Portugal deveria diminuir, revelando assim uma oposição à imigração que se encontra latente na perceção coletiva. Os profissionais enquanto parte integrante deste contexto sociocultural refletem as divisões sociais e os preconceitos da sociedade mais ampla, podendo as suas crenças interferir na prestação de cuidados de saúde.

41 No quadro geral da imigração e saúde, os dados apontam no sentido de uma relação complexa entre o processo migratório e o estado de saúde dos imigrantes. Por um lado, grande parte das causas de doença é atribuída a condições socioeconómicas precárias e a situações de exclusão social a que, frequentemente, os imigrantes estão expostos. No mesmo sentido, alguns estudos salientam que a instabilidade e precariedade socioeconómica e o reduzido acesso à educação e aos serviços de saúde que persiste entre a população imigrante determinam distintos níveis de exposição a situações de risco, o que influencia a sua vulnerabilidade em saúde (Wolffers et al., 2003; Scheppers et al., 2006). Por outro lado, os profissionais também consideram que os riscos para a saúde a que os imigrantes estão expostos são análogos aos da restante população (Inquérito Nacional de Saúde 2005-2006, 2009; Fonseca e Silva, 2010).

42 As opiniões dos profissionais apontam para a noção de que as particularidades inerentes ao estado de saúde dos imigrantes se encontram associadas a uma panóplia de fatores como o contexto ambiental do país de origem, o processo migratório, a ausência de mecanismos de suporte social e emocional, as práticas culturais e religiosas, as condições socioeconómicas, as dificuldades na procura de cuidados de saúde e na obtenção de medicamentos e tratamentos prescritos (Ingleby et al., 2005; Dias e Gonçalves, 2007; Dias, Rodrigues et al., 2010; Fonseca e Silva, 2010). Tal revela a necessidade de repensar a complexa teia de fatores que compõem a vulnerabilidade em saúde da população imigrante, bem como as suas consequências no bem-estar.

43 No contexto da saúde pública, embora os dados quantitativos indiquem que a maioria dos profissionais considera que a doença nos imigrantes não constitui um risco para a saúde pública, alguns médicos e enfermeiros, nos grupos focais, referem que esse risco existe. Vários participantes salientaram que, frequentemente, a perceção dos profissionais sobre os problemas de saúde é expressão dos preconceitos e estereótipos que estes atribuem a determinados grupos de imigrantes. A noção de que os imigrantes são potenciais portadores de doenças que representam uma ameaça à sociedade pode, de facto, colocar esta população perante situaçães de discriminação e estigmatização que se estendem ao contexto da prestação de cuidados. Estudos anteriores sugerem a existência de episódios de discriminação nos serviços de saúde, o que se pode traduzir na presença de atitudes negativas face a utentes imigrantes, com implicações nos cuidados de saúde prestados e na procura dos serviços de saúde (Dias e Rocha, 2009; Priebe et al., 2011). 

violência, o que, de acordo com Lages et al. (2006), se encontra ancorado na atribuição de estereótipos negativos a esta população, favorecendo situações de tratamento diferencial com implicações (in)diretas nos estados de saúde. A perceção de que os imigrantes apresentam uma atitude mais reivindicativa do que a restante população é explicada por Campayo et al. (2006) como um possível mecanismo de defesa criado pelos profissionais face à perceção, veiculada pelos próprios imigrantes, de que são alvo de discriminação nos serviços de saúde. ser descritas em vários estudos (Campayo et al., 2006; Michaelsen et al., 2004; Van Ryn e Fu, 2003; Priebe et al., 2011). A postura dos profissionais em relação aos imigrantes pode ser reflexo da sua incapacidade para lidar com utentes de contextos culturais distintos (Eshiett e Parry, 2003; Ingleby et al., 2005). Associada às situações de discriminação descritas pelos participantes dos grupos focais, é possível constatar o estigma que é atribuído a determinados grupos de imigrantes. A expressão subtil do preconceito que se revela através das atitudes dos profissionais pode suscitar comportamentos discriminatórios no âmbito do atendimento, cujo processo pode conduzir a uma diferencial classificação dos imigrantes, dos estados de saúde e doença, dos processos de diagnóstico e da definição de tratamentos (Campayo et al., 2006; Eshiett e Parry, 2003; Michaelsen et al., 2004; Dias et al., 2010). A coexistência de relações de poder assimétricas pode conduzir à imposição de valores e configurações de estilos de vida de acordo com o quadro de referência dos profissionais, passível de ter implicações no relacionamento entre profissionais e utentes imigrantes, bem como na continuidade de procura dos serviços de saúde (Dias e Rocha, 2009; Dias et al., 2010).

processos de discriminação e estigmatização também assentam numa dimensão histórica, sendo a experiência colonial um marco importante para compreender a formação das representações e das atitudes face aos imigrantes (Vermeulen, 2001; Bastos, 1998). O impacto da história colonial portuguesa na perceção coletiva sobre a imigração e os imigrantes pode constituir um importante fator explicativo das atitudes ambivalentes que emergiram a partir dos dados, traduzindo uma clivagem entre o plano dos discursos e das práticas dos profissionais. Os discursos podem igualmente ser reflexo da conjuntura atual, que assenta na preocupação em dar resposta a situações de discriminação, na promoção da cidadania, e na integração dos imigrantes nos vários setores da sociedade, nomeadamente, na área da saúde, do trabalho e da educação. De facto, a evolução das políticas de imigração adotadas nos últimos anos em Portugal é reflexo dos esforços realizados em prol da sua integração (Fonseca et al., 2009; Bäckström, 2009). Especificamente no domínio da saúde, a complexidade intrínseca a este processo de integração constitui assim um desafio ao desenvolvimento da cooperação mútua entre a população imigrante e os profissionais de saúde.

No que se refere aos desafios que os profissionais enfrentam no contexto da prestação de cuidados de saúde aos imigrantes, a maioria dos participantes dos grupos focais apontou para dificuldades de comunicação devido a diferenças linguísticas. A barreira da língua, por um lado, dificulta o desempenho dos profissionais, nomeadamente ao nível do diagnóstico e da prescrição de tratamentos e, por outro lado, impede que o indivíduo expresse as suas necessidades em saúde e obtenha a informação necessária para aceder aos serviços (Scheppers et al., 2006; Hultsjö e Hjelm, 2005; Fonseca e Silva, 2010; Priebe et al., 2011). As respostas encontradas pelos profissionais para colmatar 
estas dificuldades, nomeadamente a comunicação noutra língua ou por gestos, a solicitação de um familiar que funcione como intérprete, o recurso a um funcionário do Centro de Saúde para tradução e a utilização dos serviços de tradução telefónica, refletem a sua preocupação em promover boas práticas no contexto da prestação de cuidados, tal como tem sido documentado (Fonseca e Silva, 2010; Priebe et al., 2011). A falta de informação sobre as práticas culturais e religiosas, descrita pelos participantes, pode criar ruturas na relação entre profissional e utente imigrante, assim como na prática clínica (Esteva et al., 2006; Harmsen et al., 2005; Priebe et al., 2011; Scheppers et al., 2006). A cultura produz uma matriz de saberes e crenças na qual se exprimem conceções particulares de saúde e doença que influenciam o reconhecimento dos sintomas e a perceção das suas causas, bem como a perceção da necessidade de procurar cuidados de saúde e o percurso terapêutico adotado (Eshiett e Parry, 2003; Fuertes e Laso, 2006). Os participantes reconheceram a influência do seu desconhecimento das especificidades culturais e religiosas na interpretação das práticas dos imigrantes que são, frequentemente, consideradas como desadequadas de acordo com o paradigma biomédico. Neste sentido, reconhece-se que a aplicação do conhecimento médico não deve ser separada do património cultural das populações (Abreu, 2003). Esta perspetiva socioconstrutiva da aprendizagem na área da saúde constitui a base para a definição de uma visão holística no âmbito da prestação de cuidados, tornando imprescindível que o desenvolvimento de competências no contexto da diversidade cultural esteja presente ao longo de todo o processo de formação.

49 A falta de prática no diagnóstico e tratamento de doenças tropicais, descrita no grupo focal com médicos como uma dificuldade na prestação de cuidados e, ainda, como um obstáculo à continuidade da utilização dos serviços, foi igualmente evidenciado por outros autores (Esteva et al., 2006; Vázquez-Villegas, 2006). Tal realça a necessidade de desenvolver protocolos específicos de encaminhamento e atuação que permitam desenvolver a cooperação entre profissionais e, em simultâneo, dar uma resposta adequada às necessidades em saúde da população imigrante.

Em termos globais, vislumbram-se atitudes menos positivas entre os administrativos, constituindo o grupo de participantes que mais frequentemente relata experiências de contacto negativas com utentes imigrantes. As diferenças entre grupos profissionais podem ser explicadas com base no papel determinante que as experiências e funções específicas, bem como os fatores sociodemográficos, desempenham na definição das perceções e atitudes (Michaelsen et al., 2004). Neste sentido, os dados revelam a necessidade de configuração de um corpo clínico detentor de uma visão crítica e autoreflexiva e de estimular o desenvolvimento de formação adaptada aos diferentes perfis profissionais.

51 Por fim, os resultados obtidos devem ser interpretados tendo em conta as limitações das opções metodológicas. No estudo quantitativo, a informação recolhida através de questionários com perguntas fechadas é de âmbito restrito, traduzindo-se em dados estatísticos que nos fornecem respostas de natureza relativamente superficial (Giddens, 2009). No entanto, a representatividade da amostra, bem como a elevada taxa de resposta (80\%), constituem pontos fortes deste estudo.

Os dados de natureza qualitativa superam as lacunas produzidas pelo tipo de perguntas do questionário, proporcionando uma visão mais aprofundada e pormenorizada face ao tema em análise. No entanto, a dinâmica de grupo, inerente aos grupos focais, pode 
inibir a expressão individual de opiniões e experiências que não são consistentes com as da maioria do grupo. Acrescenta-se ainda o facto de que, possivelmente, o número de grupos focais não foi suficiente de modo a esgotar as possíveis perceções sobre a temática.

\section{Considerações finais}

Embora os profissionais de saúde revelem atitudes tendencialmente positivas perante os imigrantes, persistem algumas dificuldades e obstáculos no contexto da prestação de cuidados de saúde a estas populações que remetem para a persistência de situações de discriminação com implicações na qualidade dos cuidados de saúde prestados e nos percursos terapêuticos adotados.

Os profissionais constituem um elemento-chave no contexto da relação profissional de saúde-utente e, neste sentido, assumem um papel fulcral no âmbito da diminuição das desigualdades e dificuldades encontradas pelos imigrantes na procura de cuidados (Horner et al., 2004; Ingleby et al., 2005). A postura dos profissionais é um fator determinante para o (in)sucesso da prestação de cuidados e reflete a (in)capacidade dos mesmos para gerir os aspetos inerentes às especificidades culturais (Eshiett e Parry, 2003; Navarrete et al., 2009). Neste sentido, a realidade atual tornou a aquisição de competências na área da diversidade cultural um ímpeto incontornável para os profissionais. A premência da produção de um corpo de saberes e instrumentos que possam contribuir para o entendimento dos aspetos associados à diversidade cultural torna-se cada vez mais importante nos vários sectores da sociedade, em particular no sector da saúde dado o seu papel no bem-estar e na definição do estado de saúde das populações, bem como no próprio processo de integração (Machado, 2007; Ingleby et al., 2005).

O processo de capacitação dos profissionais para lidar com a diversidade cultural implica, em primeiro lugar, contemplar o papel dos fatores sociais e políticos enquanto dimensões imprescindíveis para a integração da dimensão cultural na prestação de cuidados (Pussetti et al., 2009). Neste contexto, a formação dos profissionais assume particular relevo no âmbito do desenvolvimento de competências e, em simultâneo, na resposta às questões associadas aos processos de discriminação e estigmatização. 0 conhecimento e a compreensão das particularidades socioculturais, em conjunto com a mediação intercultural, constituem assim aspetos fulcrais na dinamização do diálogo entre profissionais e utentes, o que se vai refletir na estruturação de intervenções informadas e adequadas e contribuir para a criação de um espaço terapêutico "culturalmente sensível" dotado de uma maior capacidade para gerir a crescente diversidade cultural e étnica que caracteriza a realidade atual.

\section{BIBLIOGRAFIA}

ABREU, W. C. (2003), Saúde, doença e diversidade cultural, Lisboa, Instituto Piaget. 
BÄCKSTRÖM, B. (2009), Saúde e Imigrantes - As representações e as Práticas sobre a Saúde e a Doença na Comunidade Cabo-Verdiana em Lisboa, Lisboa, ACIDI.

BARDIN, L. (2007), Análise de Conteúdo, Lisboa, Edições 70.

BASTOS, C. (1998), “Tristes trópicos e alegres luso-tropicalismos: das notas de viagem em LéviStrauss e Gilberto Freyre”, Análise Social, XXXIII(146-147), pp. 415-32.

BETANCOURT, J., et al. (2003), "Defining Cultural Competence: A Practical Framework for Addressing Racial/Ethnic Disparities in Health and Healthcare", Public Health Reports, 118, pp. 293-302.

CAMPAYO, J. G., et al. (2006), "Attitudes of Spanish doctors towards immigrant patients: an opinion survey”, Actas Españolas de Psiquiatria, 34(6), pp. 371-76.

DAVIES, A. A., et al. (2009), Migration: a social determinant of the health of migrants, Geneva, IOM Immigration Health Department.

DIAS, S. e A. Gonçalves (2007), “Migração e Saúde”, Migrações, 1, pp. 15-26.

DIAS, S. e C. Rocha (2009), Saúde Sexual e Reprodutiva de Mulheres Imigrantes Africanas e Brasileiras: Um estudo qualitativo, Lisboa, ACIDI.

DIAS, S., et al. (2010), “Immigrant women's perceptions and experiences of health care services: Insights from a focus group study", Journal of Public Health, 18, pp. 489-96.

DIAS, S.; R. Rodrigues, et al. (2010), "Procura de cuidados e acesso aos serviços de saúde em comunidades imigrantes: Um estudo com imigrantes e profissionais de saúde", Arquivos de Medicina, 24(6), pp. 253-59.

ESHIETT, M. U. A. e E. H. O. Parry (2003), “Migrants and health: a cultural dilemma”, Clinical Medicine, 3(3), pp. 229-31.

ESTEVA, M., et al. (2006), "Percepción de las dificultades en la atención sanitaria al inmigrante económico en medicina de família", Aten Primária, 37(3), pp. 154-59.

FONSECA, M. L., et al. (2009), MIGHEALTHNET - Relatório sobre o Estado da Arte em Portugal, Lisboa, Centro de Estudos Geográficos.

FONSECA, M. L. e S. Silva (2010), Saúde e Imigração: Utentes e Serviços na Área de Influência do Centro de Saúde da Graça, Lisboa, ACIDI.

FUERTES, C. e M. A. Laso (2006), “El inmigrante en la consulta de atención primaria”, An. Sist. Sanit. Navar., 29(1), pp. 9-25.

GIDDENS, A. (2009), Sociologia, Lisboa, Fundação Calouste Gulbenkian.

HARMSEN, H., et al. (2005), "The effect of educational intervention on intercultural communication: Results from a randomised controlled trial”, British Journal of General Practice, 55, pp. 343-350.

HORNER, R. D., et al. (2004), “Changing Healthcare Professionals' Behaviors to Eliminate Disparities in Healthcare: What do we know? How might we proceed?", The American Journal of Managed Care, 10, pp. 12-19.

HULTSJÖ, S. e K. Hjelm (2005), “Immigrants in emergency care: Swedish health care staff's experiences”, International Nursing Review, 52, pp. 276-85.

INE e INSA, Inquérito Nacional de Saúde 2005-2006 (2009), Lisboa, INE/INSA. 
INGLEBY, D., et al. (2005), “The Role of Health in Integration”, in M. L. Fonseca e J. Malheiros (coords.), Social Integration and Mobility: Education, Housing and Health - Imiscoe Cluster B5, State of the Art Report, Lisboa, Centro de Estudos Geográficos: Estudos para o Planeamento Regional e Urbano 67, pp. 101-37.

KRUEGER, R. e M. A. Casey (2000), Focus groups: A practical guide for applied Research, Thousand Oaks, Sage Publications.

LAGES, M. F., et al. (2006), Os imigrantes e a população portuguesa - Imagens recíprocas, Análise de duas sondagens, Lisboa, ACIDI.

LESSARD-HÉRBERT, M., et al. (1994), Investigação Qualitativa - Fundamentos e Práticas, Lisboa, Instituto Piaget, pp. 29-62.

MACHADO, F. L. (2007), “Migrações, saúde e doença - que investigação em Portugal?”, Migrações, 1, pp. 201-3.

MICHAELSEN, J. J., et al. (2004), "Health professionals' knowledge, attitudes and experiences in relation to immigrant patients: a questionnaire study at a Danish Hospital", Scandinavian Journal of Public Health, 32, pp. 287-95.

MORGAN, D. (2001), "Focus Group Interviewing”, in J. F. Gubrium e J. A. Holstein (eds.), Handbook of interview research: context and method, Thousand Oaks, CA, Sage Publications.

NAVARRETE, M., et al. (2009), “Necesidades de los Profesionales de Salud en la Atención a la Población Inmigrante", Gaceta Sanitária, 23(5), pp. 396-402.

PEIRO, M. J. e R. Benedict (2009), Migration Health: Better health for All in Europe. Final Report Assisting Migrants and Communities (AMAC): Analysis of Social Determinants of Health and Health Inequalities, Bruxelas, IOM.

PRIEBE, S., et al. (2011), "Good practice in health care for migrants: views and experiences of care professionals in 16 European countries”, BMC Public Health, 11, pp. 187, doi:

10.1186/1471-2458-11-187.

PUSSETTI, C., et al. (2009), Migrantes e Saúde Mental. A Construção da Competência Cultural, Lisboa, ACIDI.

SCHEPPERS, E., et al. (2006), "Potential barriers to the use of health services among ethnic minorities: a review”, Family Practice, 23(3), pp. 325-48.

VAN RYN, M. e J. Burke (2000), "The effect of patient race and socio-economic status on physicians' perceptions of patients”, Social Science and Medicine, 50, pp. 813-828.

VAN RYN, M. e S. S. Fu (2003), "Paved With Good Intentions: Do Public Health and Human Service Providers Contribute to Racial/Ethnic Disparities in Health?", American Journal of Public Health, 93(2), pp. 248-55.

VÁZQUEZ-VILLEGAS, J. (2006), “Inmigración y salud: ¿un nuevo modelo de atención primaria para un nuevo modelo de sociedad?", Aten Primaria, 37, pp. 249-50.

VERMEULEN, H. (2001), Imigração, integração e a dimensão política da cultura, Lisboa, Edições Colibri. WOLFFERS, I., et al. (2003), “Migration, human rights and health”, Lancet, 362 (9400), pp. 2019-20. 


\section{NOTAS}

1. Este estudo foi financiado pela Fundação para a Ciência e a Tecnologia (IME/SAUESA/ 81760/2006). Os autores agradecem a colaboração de todos os participantes do estudo e os membros da equipa do projeto, especialmente Helena Cargaleiro, Maria do Rosário Horta, Fernanda Silva, António Silva, Mário Carreira, Violeta Alarcão, Miguel Lemos, Maria Cortes e Ana Gama.

\section{RESUMOS}

A migração, enquanto fenómeno social complexo, constitui um importante desafio nas dinâmicas dos serviços de saúde e na prestação de cuidados de saúde nos países de acolhimento. Neste estudo pretende-se descrever as perceções e atitudes dos profissionais de saúde no âmbito da prestação de cuidados a imigrantes e compreender os desafios que enfrentam neste contexto. Para a concretização dos objetivos propostos realizaram-se dois estudos, um estudo quantitativo através do recurso a um inquérito por questionário e um estudo qualitativo através da realização de grupos focais. Os dados obtidos apontam para que embora os profissionais revelem atitudes tendencialmente positivas perante os imigrantes, persistem algumas dificuldades e obstáculos no contexto da prestação de cuidados a estas populações. Neste contexto, os resultados sublinham a importância da criação de um espaço terapêutico "culturalmente sensível" através do incremento de formação adaptada aos diferentes perfis profissionais.

The complex phenomenon of migration has been a concern for public health, constituting a major challenge in the dynamics of health services and the provision of health care in host countries. This study aims to explore perceptions and attitudes of health professionals, and better understand the challenges faced in the context of health provision to immigrants. Two studies were conducted with health professionals, a quantitative study with a self-administered questionnaire and a qualitative study using focus groups. Data obtained indicate that albeit health professionals tend to show positive attitudes toward immigrants, some difficulties and obstacles persist in provision of health care to migrant populations. In this context, empirical data highlights the importance of developing a "culturally sensitive" therapeutic space by providing training on cultural diversity adapted to the different professionals' profiles.

\section{ÍNDICE}

Keywords: health professionals, attitudes, provision of health care, immigrants

Palavras-chave: profissionais de saúde, atitudes, prestação de cuidados de saúde, imigrantes

\section{AUTORES}

\section{RITA RODRIGUES}

Instituto de Higiene e Medicina Tropical, Universidade Nova de Lisboa \& Centro em Rede de Investigação em Antropologia (CRIA) (rodrigues.arita@gmail.com) 


\section{SÓNIA DIAS}

Unidade de Saúde Pública Internacional, Instituto de Higiene e Medicina Tropical, Universidade Nova de Lisboa \& Centro de Malária e Doenças Tropicais (CMDT) (smfdias@yahoo.com) 